\title{
Characterization of VLSI Processing Defects Using STEM-EELS Tomography
}

\author{
Frieder H. Baumann ${ }^{1}$, John Miller ${ }^{1}$, Bryan Rhoads ${ }^{1}$, Anne Friedman ${ }^{1}$, Bianzhu Fu ${ }^{2}$ \\ ${ }^{1 .}$ Globalfoundries, 2070 Route 52, Hopewell Junction, NY 12533 \\ ${ }^{2}$ Globalfoundries, 400 Stone Break Rd Extension, Malta, NY 12020, USA
}

Modern Very Large Scale Integrated (VLSI) circuits contain devices which are inherently 3D in nature (tri-gate transistors, fin-FETs) and which approach nanometer dimensions. Characterization of these devices and their defects requires chemically sensitive 3D techniques with near atomic resolution. Chemically sensitive electron tomography using EDX (electron dispersive X-ray) has been demonstrated to be able to successfully deliver 3D information with nanometer resolution, see e. g. [1][2].

We employed EELS (Electron Energy Loss Spectroscopy) tomography to characterize defects in the early processing stages of our Fin FET (fin Field effect transistor). Although the EELS technique is conceptually more difficult than the above mentioned EDX method, EELS is able to differentiate between different valence states of a chemical substance. In the case of silicon, EELS is able to distinguish between Si atoms which are unbonded (i. e. poly-Si, single crystal $\mathrm{Si}$ ) and chemically bonded $\mathrm{Si}$ in oxide or nitride. For pure $\mathrm{Si}$, the $\mathrm{Si}-\mathrm{L}_{2,3}$ edge onset is at $99 \mathrm{eV}$, while the $\mathrm{Si}-\mathrm{L}_{2,3}$ edge appears at $106 \mathrm{eV}$ for $\mathrm{SiO}_{2}$ [3]. Thus, monitoring the EELS signal between 99 and $106 \mathrm{eV}$ will produce signal from pure Si only.

We investigates nano-scale lines of $\alpha$-Si (amorphous silicon) which are used to build our Si devices. These a-Si lines are fabricated by depositing an amorphous Si blanket layer on the wafer, and subsequently etch out unwanted material. The $\alpha$-Si lines can serve as etch masks, moldings, mandrels, or casts for the electronic devices build in a complex fabrication process. Any defect in one of these $\alpha$-Si lines, however, may translate later into an electrical defect of one of the devices. Thus, understanding and eliminating the defects is of paramount importance.

EDX (FEI Osiris) mapping of a typical defect between two a-Si lines is shown in Fig. 1. A faint Si signal can be seen on the right shoulder of the left line. It is believed that a micro-masking effect during the etch left some $\alpha$-Si standing between the lines. The O-map indicates that a thin oxide layer seems to cover the top of the defect. All in all, imaging and x-ray mapping is barely able to characterize the defect.

The result of the STEM-EELS tomography is shown in Fig. 2, clearly showing a block of silicon left standing between the $\alpha$-Si lines. In addition, the oxide cap on top of the un-etched Si block can easily be spotted. For the tomography, a total of 23 EELS maps, with tilt angle ranging from $-55^{\circ}$ to $+55^{\circ}$ in $5^{\circ}$ steps, were acquired in a probe-corrected FEI Titan microscope operating at $200 \mathrm{kV}$. Each map consisted of 100x100 pixels and required about 5 min to acquire. Total acquisition time of the data was $\sim 5$ hours. Data analysis consisted of noise filtering using principal component analysis for both the Si and the Oxygen edge, followed by reconstruction using the SIRT algorithm for Si and weighted back-projection for $\mathrm{O}$ (Inspect3D, FEI). For visualization, the Amira software suite was employed using segmentation.

In conclusion, we show that STEM-EELS tomography can be used to characterize defects in modern VLSI technologies on the nano-meter scale. Since the EELS signal is sensitive to the valence state of many materials, EELS tomography is shown to be able to distinguish, in 3D on the nano-meter scale, between atoms (here $\mathrm{Si}$ ) in different environments (a-Si, nitride, oxide). 


\section{References:}

[1] A Genç et al, Microscopy and Analysis 116, 23-25, (2012)

[2] K. Lepinay et al, Micron. 47: 43-9, (2013)

[3] M. S. M. Saifullah et al, Electron Microscopy 96, vol 2, (Committee of European Societies of Microscopy, Brussels, ) p 123-124, (1998)
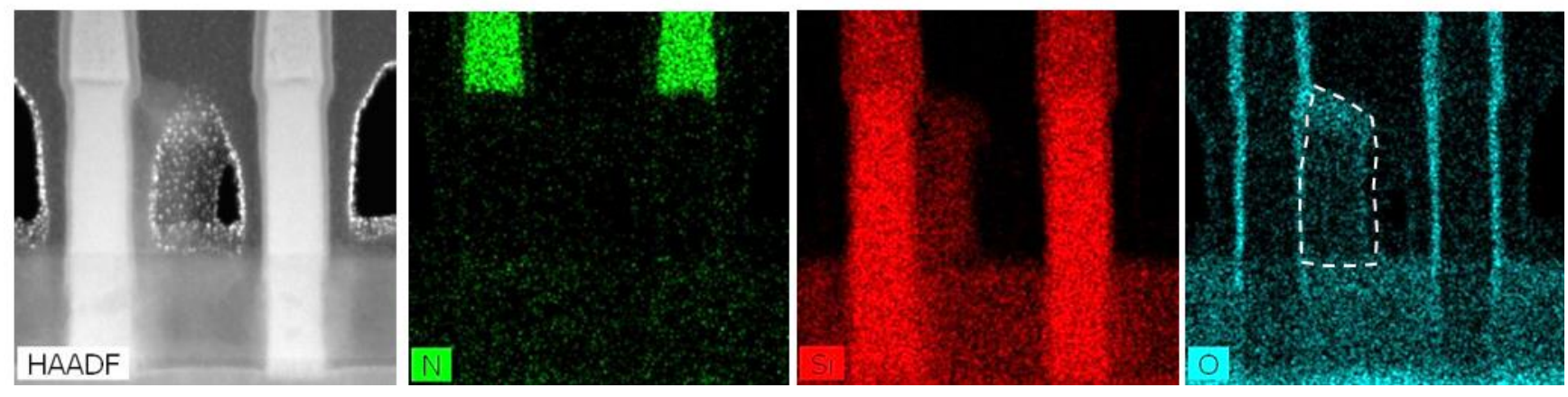

Figure 1. X-ray mapping of defect between two a-Si lines. The Si map (red) shows a faint signal at the right shoulder of the left line. The perceived outline of the defect is shown in the O map. Imaging and $\mathrm{X}$-ray mapping are barely able to give insight to the nature of the defect.
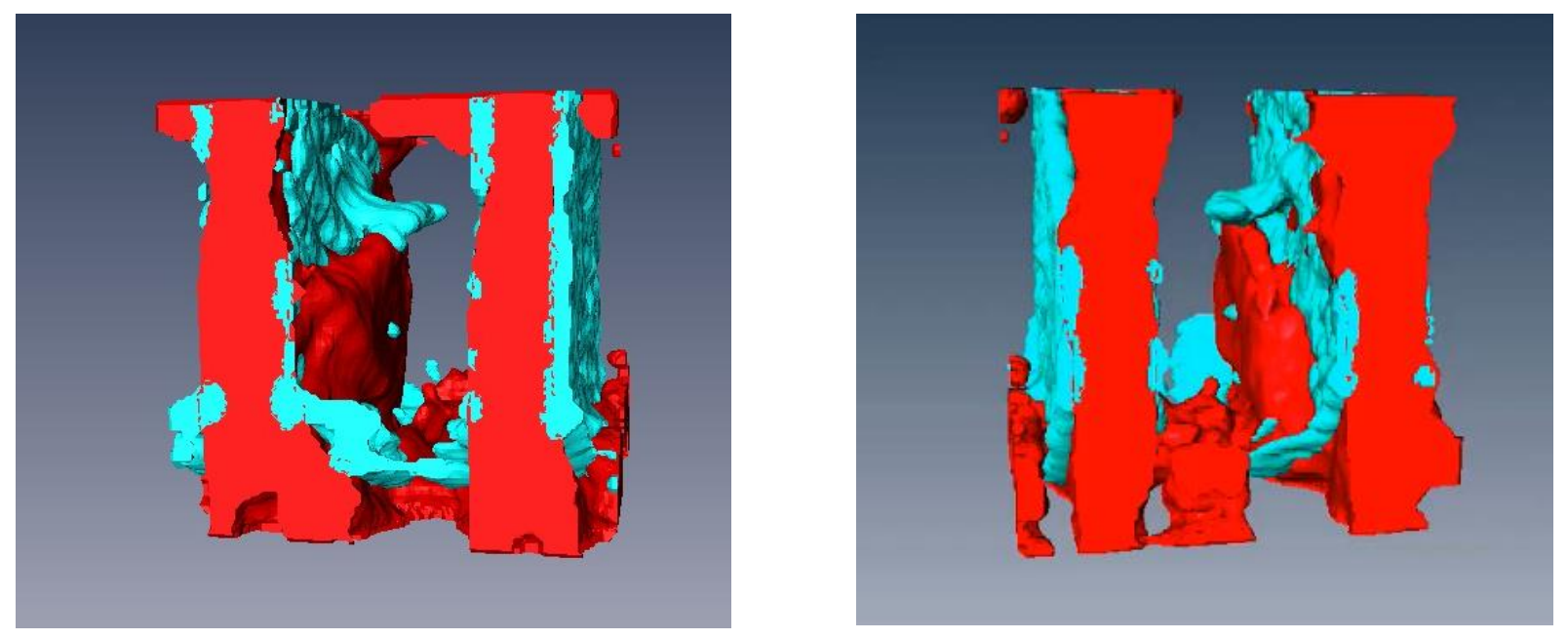

Figure 2. Two snapshots of the micro-mask defect, reconstructed from a total of 23 EELS maps. The reconstruction shows Oxygen (blue) and unbound $\mathrm{Si}$ (red). The oxide cap on top of the un-etched Si block is clearly resolved, as well as a thin $\mathrm{SiO} 2$ layer on the sides of the $\alpha$-Si lines. 